\title{
Ansiedad ante los exámenes en los estudiantes del Centro Preuniversitario - UNMSM ciclo 2012-I
}

\author{
Test anxiety in students of CEPRE - UNMSM cycle 2012-I \\ Hilda Chávez C. ${ }^{1}$, José Chávez Z., Eduardo Ruelas S., Miguel Gómez B. \\ Universidad Nacional Mayor de San Marcos, Lima, Perú \\ (RECIBIDO 16-09 2014, ACEPTADO 25-11-2014)
}

\begin{abstract}
RESUMEN
El presente estudio descriptivo tiene como objetivo abordar las reacciones de ansiedad de los estudiantes del CEPRE-UNMSM, Ciclo Ordinario 2012 - I. ante los exámenes se utilizó la escala de ansiedad ante los exámenes - IDASE (Bauermeister, Collazo \& Spielberger, 1982), que evalúa los factores emocionalidad y preocupación. Se aplicó a 800 estudiantes haciendo uso de un muestreo no probabilístico intencional. Los resultados obtenidos indicaron que el $27 \%$ de los estudiantes presenta un nivel alto de ansiedad ante los exámenes, mientras que el $33.38 \%$ y $39.63 \%$ posee un nivel bajo y moderado, respectivamente. Asimismo, existe un mayor nivel de ansiedad en el sexo femenino (17.13\%) sobre el masculino (9.88\%), así como también, mayor ansiedad en los postulantes a Medicina Humana, Derechoy Administración.
\end{abstract}

Palabras clave: Ansiedad ante los exámenes, emocionalidad, preocupación, estudiantes del CEPRE-UNMSM.

\begin{abstract}
The present paper had the purpose of exploring test anxiety reactions in students of the CEPRE-UNMSM 2012 - I Cycle. The instrument used to gather the data was the Test Anxiety Inventory (Bauermeister, Collazo \& Spielberger, 1982) that evaluates emotionality and preoccupation factors. The population was constituted by 800 students using an intentional non-probability sampling. The results indicate that $27 \%$ of the students present a high level of test anxiety, whereas $33.38 \%$ of the students have a low level test anxiety and $39.63 \%$, a moderate level. In addition to that, regarding the gender, female students had a higher level of anxiety $(17,13 \%)$ over male students $(9,88 \%)$. Moreover, there was higher anxiety level in candidates of the following careers: Medicine, Law and Management.
\end{abstract}

Keywords: Test anxiety, emotionality, preoccupation, CEPRE-UNMSM 2012-I Cycle students.

* E-mail: hchavezch@hotmail.com 


\section{INTRODUCCIÓN}

La Unidad Psicopedagógica del CEPRE-UNMSM durante su labor de orientación y consejería psicológica ha encontrado que los alumnos del CEPRE presentan una problemática variada, la cual afecta su rendimiento académico. Es así que -entre la ansiedad, depresión, autocontrol emocional, técnicas de estudio inadecuadas, dudas vocacionales, problemas personales, problemas familiares, presión de los padres, baja autoestima y demás constituyen algunos de los principales problemas que demandan mayor atención y, en consecuencia, resultan afectando directamente el rendimiento académico.

A medida que se acercan los exámenes, la ansiedad de los alumnos comienza a incrementarse; se hace evidente que ella se manifiesta básicamente en las dimensiones emocionalidad y preocupación y genera en muchos casos alteraciones psicosomáticas, que, antes y durante los exámenes, afectan el rendimiento académico. Asimismo, se evidencia que muchos de los estudiantes que solicitan atención psicopedagógica poseen conocimientos bien cimentados, sin embargo, al momento de rendir sus exámenes, obtienen resultados por debajo de su nivel académico, la Unidad Psicopedagógica se ha plantea do como objetivo investigar el nivel de ansiedad ante los exámenes que poseen los alumnos del CEPRE-UNMSM, ciclo ordinario 2012-I, para lo cual se ha utilizado como instrumento el Inventario de autoevaluación de ansiedad ante exámenes-IDASE, elaborado por Bauermeister, Collazo \& Spielberger en 1982, cuyos puntajes totales directos, así como también las dimensiones de emocionalidad y preocupación, fueron baremados por Aliaga, Ponce, Bernaola y Pecho (Lima, 2001).

Para efectuar esta investigación se ha evaluado con el IDASE a una muestra de ochocientos estudiantes $(n=800)$, estableciéndose los niveles de ansiedad, tanto en forma general como por las dimensiones correspondientes, considerando para dicho análisis la variable sexo.

Los resultados obtenidos se enriquecen al ser analizados no solo desde el punto de vista cuantitativo, sino también comparándolos con la estadística de la Unidad Psicopedagógica y la apreciación de sus integrantes.

\section{Planteamiento del problema}

\section{Problema general}

- ¿Cuál es el nivel de ansiedad, ante los exámenes, que poseen los alumnos del CEPREUNMSM, ciclo ordinario 2012 - I?

\section{Problemas específicos}

- ¿Qué niveles de ansiedad ante los exámenes, en el factor emocionalidad, presentan los estudiantes del CEPRE-UNMSM, ciclo ordinario 2012-I?

- ¿Cuáles son los niveles de ansiedad ante los exámenes, en el factor preocupación, que presentan los estudiantes del CEPRE-UNMSM, ciclo ordinario 2012-I? 


\section{Objetivos}

\section{Objetivo general}

- Conocer el nivel de ansiedad, ante los exámenes, que poseen los alumnos del CEPREUNMSM del ciclo ordinario 2012 - I.

\section{Objetivos específicos}

- Determinar los niveles de ansiedad ante los exámenes, en el factor emocionalidad, de los estudiantes del CEPRE-UNMSM, Ciclo Ordinario 2012 - I.

- Determinar los niveles de ansiedad ante los exámenes, en el factor preocupación, de los estudiantes del CEPRE-UNMSM, Ciclo Ordinario 2012 - I.

\section{Marco teórico}

\section{Antecedentes}

En el ámbito nacional e internacional encontramos diversos estudios, tanto descriptivos como correlacionales y explicativos sobre la ansiedad ante los exámenes, relacionándola con diversas variables. Entre los principales estudios, sobre nuestra variable de interés, tenemos los siguientes:

Grandis (Córdoba, 2009) elaboró y validó un cuestionario de ansiedad frente a los exámenes, en cuya estructura se medían tres factores: cognitivo, fisiológico y de evitación. La muestra estuvo conformada por 1368 estudiantes universitarios. Los resultados según géneroy facultad de procedencia evidenciaron que, con respecto a la variable género, el mayor nivel de ansiedad se encuentra en los estudiantes del género femenino; y, con respecto a la Facultad de procedencia, el mayor nivel de ansiedad fue encontrado en los estudiantes de la facultad de Ciencias Humanas, mientras que el menornivel de ansiedad fue reportado por los estudiantes de la Facultad de Ingeniería.

Reyes (Lima, 2003) estableció la relación entre rendimiento académico, ansiedad ante los exámenes, rasgos de personalidad, autoconcepto y asertividad en una muestra de 62 estudiantes del primer año de Psicología de la UNMSM. Utilizando como instrumentos: el inventario de ansiedad ante los exámenes, el cuestionario de personalidad 16 pf de cattell, el cuestionario de autoconcepto forma A y el autoinforme de conducta asertiva. Los resultados indican que el mejor predictor del rendimiento académico es el autoconcepto académico.

Piemontesi, Heredia y Furián (2009) estudiaron la relación entre la ansiedad ante los exámenes, estrategias de afrontamiento frente a un examen importante, autoeficacia para el aprendizaje autorregulado y el rendimiento académico en una muestra de 210 estudiantes universitarios. Utilizando como instrumentos: el inventario alemán de ansiedad ante los exámenes (GTAI-A), medida de afrontamiento ante la ansiedad e incertidumbre pre-examen: (COPEAU), escala de autoeficacia para el aprendizaje autorregulado (SELF-A). Los resultados indican que la preocupación ante los exámenes (dimensión de la ansiedad ante los exámenes) correlaciona positivamente con orientación a la tarea y negativamente con evitación. 
Chávez (2004) estudió los efectos de un programa de intervención, con un diseño antes-después como parte de un modelo educativo innovador aplicado en la Universidad de Colima en un grupo de estudiantes de Psicología, orientado a probar los efectos de este programa en la ansiedad ante la evaluación. Los resultados proporcionan diferencias significativas entre los puntajes promedio-tanto grupales como individuales- en ansiedad de los estudiantes. Evaluadas con el Inventario de Situaciones y Respuestas de Ansiedad (ISRA).

Gamboa (2010) estudió los efectos de un programa de biodanza en un grupo de 48 estudiantes de Psicología de la Universidad Nacional Federico Villarreal, quienes presentaban síntomas ansiosos. Utilizó el Inventario de Ansiedad Rasgo-Estado de Spielberger (IDARE) antes y después de aplicar el programa de biodanza. Los resultados indican una disminución del nivel de ansiedad estado del $46 \%$ de estudiantes.

Valero (1999) valida el cuestionario CAEX para evaluar la ansiedad ante los exámenes, utilizando la técnica Likert recoge información descriptiva sobre respuestas motoras, verbales, cognitivas y fisiológicas que usualmente acompañan las situaciones de exámenes. Los resultados indican diferencias de género en relación con la ansiedad ante los exámenes.

\section{Ansiedad ante los exámenes}

La ansiedad ante los exámenes es considerada, según el DSM IV-TR, como un trastorno de ansiedad generalizada, caracterizada por la presencia de altos niveles de ansiedad y preocupación duradera ante una gran cantidad de estímulos y situaciones; percibidas por el sujeto como amenazas y que no está en condiciones de afrontar.

EI CIE 10 considera que la ansiedad ante los exámenes es una fobia específica, y es restringida a situaciones muy específicas. Hodapp, Glazman y Laux, en 1995, definen a la ansiedad frente a los exámenes como un rasgo especifico situacional, caracterizado por la predisposición a reaccionar con elevada ansiedad en contextos que se relacionan con el rendimiento.

\section{MÉTODO}

\section{Población}

Constituida por 5880 estudiantes distribuidos en las diferentes sedes del CEPRE UNMSM, ciclo ordinario 2012 - I.

\section{Muestra}

La muestra está conformada por 800 estudiantes del CEPRE - UNMSM del ciclo ordinario 2012 - I, que reciben clases en 42 aulas, ubicadas en los locales de, 
Santa Beatriz ( $n=622)$, jirón Callao $(n=91)$ y en las diferentes facultades de la Ciudad Universitaria ( $n=87$ ); Se tomaron 374 varones (equivalentes al $46.75 \%$ de la muestra) 426 mujeres (equivalentes al 53.25\% de la muestra), cuyas edades fluctúan entre los 14 y 32 años, una mayor concentración de estudiantes entre las edades de 15 y 22 años ( $n=773$ estudiantes). Postulan a 78 carreras profesionales, y las carreras de mayor demanda son las de Medicina, Derecho y Administración.

\section{Instrumentos}

Para desarrollar esta investigación se ha utilizado el instrumento cuyas características se detallan a continuación:

Nombre : : Inventario de Autoevaluación sobre Ansiedad ante los exámenes - IDASE.

Objetivo : Medir la predisposición de los estudiantes a reaccionar con estados de ansiedad y respuestas de preocupación ante situaciones evaluativas.

Autores : Bauermeister, Collazo y Spielberger.

Baremos : Aliaga, Ponce, Bernaola y Pecho (Lima, 2001).

Aplicación : Individual y colectiva, 11 años en adelante.

Duración : :15 minutos aproximadamente.

Confiabilidad : $r=0.60 \mathrm{p}<0.01$ Test - Retest.

\section{Procedimiento}

Planificación del proyecto de investigación.

Recopilación de datos (estado del arte).

Diseño del marco teórico.

Selección del instrumento para recoger datos.

Diseño y computarización para la calificación del IDASE.

Aplicación del IDASE a una muestra de 800 estudiantes del CEPRE-UNMSM.

Procesamiento y análisis estadístico, utilizando el MS Excel y el Software

Estadístico SPSS versión 19.

Redacción del informe.

Presentación del informe.

Propuesta de acciones correctivas. 


\section{RESULTADOS}

En forma general los resultados, según el diagnóstico de ansiedad, nos indica que el $27 \%$ de estudiantes presentan ansiedad alta ante los exámenes, mientras que el $39.63 \%$ tiene ansiedad moderada y el $33.38 \%$ presenta ansiedad baja ante los exámenes, tal como puede apreciarse en la tabla 1.

Tabla 1. Distribución de los sujetos según nivel de ansiedad.

\begin{tabular}{lcc}
\hline Diagnóstico & Total & Porcentaje \\
\cline { 2 - 3 } Ansiedad Alta & 216 & 27.00 \\
Ansiedad Moderada & 317 & 39.63 \\
Ansiedad Baja & 267 & 33.38 \\
Total general & 800 & 100.00 \\
\hline
\end{tabular}

Con la finalidad de brindar una mejor apreciación sobre estos resultados generales, presentamos gráficamente esta información (ver Figura 1), observándose con claridad el alto porcentaje de estudiantes que presentan Alta Ansiedad ante los Exámenes.

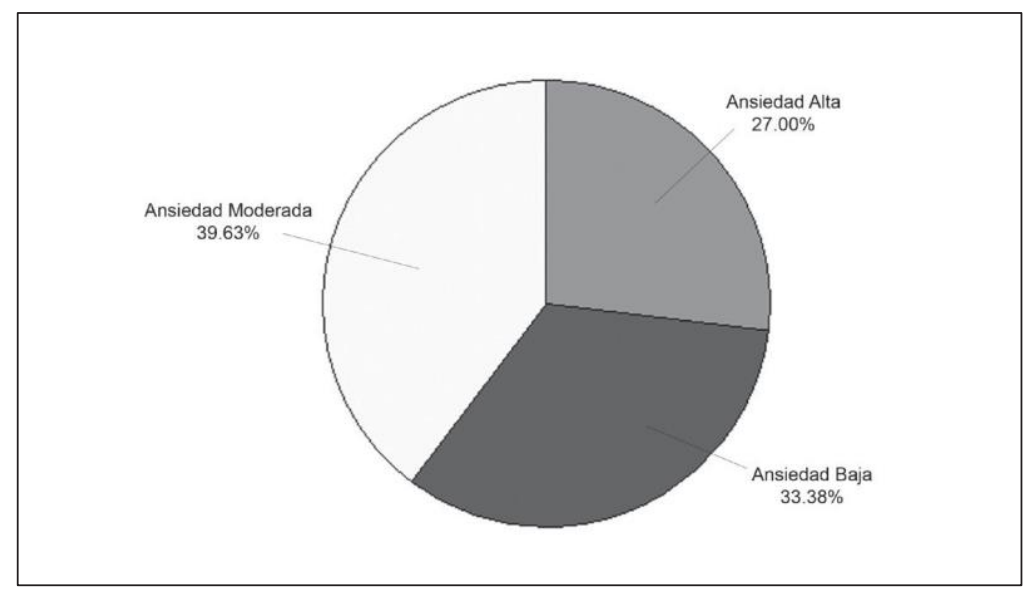

Figura 1. Ansiedad ante los exámenes. CEPRE-UNMSM. CICLO 2012 - I.

Considerando la variable sexo, encontramos que, respecto al diagnóstico ansiedad alta ante los exámenes, son las estudiantes del sexo femenino quienes presentan una mayor Ansiedad (17.13\%) que los estudiantes del sexo masculino (9.88\%). Observándose que esta predominancia del sexo femenino se mantiene enel caso del diagnóstico ansiedad moderada (22.13\% del sexo femenino y $17.50 \%$ para el sexo masculino). Sin embargo, para el diagnóstico de ansiedad baja ante los Exámenes, son los estudiantes del sexo masculino quienes, en términos porcentuales, tienen menor ansiedad ante los exámenes (19.38\% para el sexo masculino contra $14 \%$ para el sexo femenino). Tal como puede apreciarse en la tabla 2 y en la figura 2. 
Tabla 2. Distribución de los sujetos según sexo y nivel de ansiedad.

\begin{tabular}{ccccccc}
\hline & \multicolumn{2}{c}{ Masculino } & \multicolumn{2}{c}{ Femenino } & \multicolumn{2}{c}{ Total } \\
\hline Ansiedad & $\mathrm{n}$ & Porcentaje & $\mathrm{n}$ & Porcentaje & $\mathrm{n}$ & Porcentaje \\
Alta & 79 & 9.88 & 137 & 17.13 & 216 & 27.00 \\
Moderada & 140 & 17.50 & 177 & 22.13 & 317 & 39.63 \\
Baja & 155 & 19.38 & 112 & 14.00 & 267 & 33.38 \\
& & Total: & & & 800 & 100.00 \\
\hline
\end{tabular}

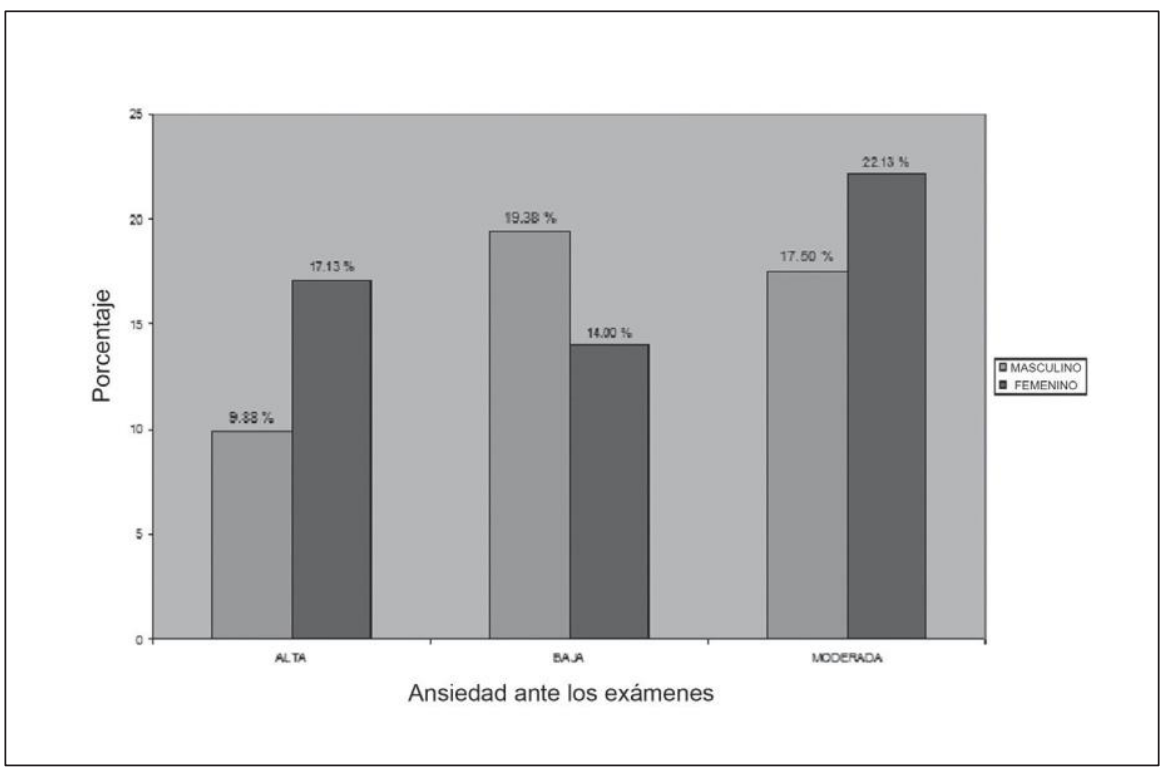

Figura 2. Ansiedad ante los exámenes según sexo.

Al procesar las ansiedad ante los exámenes por aulas, encontramos que las aulas que tienen mayor cantidad de alumnos con ansiedad alta ante los exámenes son las aulas: $27,19,06,20,37,13$ y 13 respectivamente. tal como puede apreciarse en la tabla 3 donde podemos observar claramente que en el aula 27 asistieron 58 estudiantes, de los cuales 23 fueron diagnosticados con ansiedad alta ante los exámenes, 15 con ansiedad moderada y 20 con ansiedad baja ante los exámenes (ver tabla 3), al aula 19 asistieron 46 estudiantes, de los cuales 18 fueron diagnosticados con ansiedad alta ante los exámenes, 17 con ansiedad moderada y 11 con ansiedad baja ante los exámenes (ver tabla 3), las aulas que han obtenido menor cantidad de estudiantes con ansiedad alta ante los exámenes son: $16,81,91,80$ y 26; en el caso del aula 16 asistieron 27 estudiantes, de los cuales 4 fueron diagnosticados con ansiedad alta ante los exámenes, 14 con ansiedad moderada y 9 con ansiedad baja ante los exámenes (ver tabla 3). Para este análisis no se han considerado las aulas en las que se evaluó a menos de 10 estudiantes. 
Tabla 3. Diagnóstico de ansiedad por aulas.

\begin{tabular}{|c|c|c|c|c|c|}
\hline \multirow{2}{*}{ Aula } & \multirow{2}{*}{ Datos } & \multicolumn{3}{|c|}{ Número de estudiantes con } & \multirow{2}{*}{ Total } \\
\hline & & Ansiedad alta & Ansiedad moderada & ansiedad Baja & \\
\hline 27 & $n$ & 23 & 15 & 20 & 58 \\
\hline 19 & $\mathrm{n}$ & 18 & 17 & 11 & 46 \\
\hline 06 & $\mathrm{n}$ & 15 & 11 & 5 & 31 \\
\hline 20 & $n$ & 14 & 22 & 17 & 53 \\
\hline 37 & $\mathrm{n}$ & 12 & 18 & 19 & 49 \\
\hline 13 & $\mathrm{n}$ & 12 & 7 & 8 & 27 \\
\hline 03 & $\mathrm{n}$ & 11 & 13 & 9 & 33 \\
\hline 82 & $\mathrm{n}$ & 9 & 9 & 11 & 29 \\
\hline 38 & $\mathrm{n}$ & 8 & 16 & 18 & 42 \\
\hline 14 & $\mathrm{n}$ & 8 & 15 & 8 & 31 \\
\hline 12 & $\mathrm{n}$ & 8 & 10 & 12 & 30 \\
\hline $19 \mathrm{~B}$ & $\mathrm{n}$ & 8 & 12 & 10 & 30 \\
\hline 33 & $\mathrm{n}$ & 8 & 12 & 7 & 27 \\
\hline 10 & $\mathrm{n}$ & 7 & 21 & 20 & 48 \\
\hline 07 & $\mathrm{n}$ & 7 & 15 & 12 & 34 \\
\hline 09 & $\mathrm{n}$ & 6 & 14 & 12 & 32 \\
\hline 05 & $\mathrm{n}$ & 6 & 11 & 11 & 28 \\
\hline 17 & $\mathrm{n}$ & 5 & 16 & 9 & 30 \\
\hline 16 & $\mathrm{n}$ & 4 & 14 & 9 & 27 \\
\hline 81 & $\mathrm{n}$ & 4 & 4 & 3 & 11 \\
\hline 91 & $\mathrm{n}$ & 4 & 3 & 3 & 10 \\
\hline 80 & $\mathrm{n}$ & 3 & 11 & 6 & 20 \\
\hline 26 & $\mathrm{n}$ & 2 & 13 & 17 & 32 \\
\hline 21 & $\mathrm{n}$ & 2 & 3 & 0 & 5 \\
\hline 04 & $\mathrm{n}$ & 2 & 1 & 1 & 4 \\
\hline 30 & $n$ & 2 & 0 & 0 & 2 \\
\hline 79 & $\mathrm{n}$ & 1 & 4 & 2 & 7 \\
\hline 83 & $n$ & 1 & 2 & 2 & 5 \\
\hline 84 & $\mathrm{n}$ & 1 & 1 & 0 & 2 \\
\hline 01 & $n$ & 1 & 0 & 0 & 1 \\
\hline 08 & $\mathrm{n}$ & 1 & 0 & 0 & 1 \\
\hline 15 & $n$ & 1 & 0 & 0 & 1 \\
\hline 24 & $\mathrm{n}$ & 1 & 0 & 0 & 1 \\
\hline 78 & $\mathrm{n}$ & 1 & 0 & 0 & 1 \\
\hline 28 & $\mathrm{n}$ & 0 & 2 & 1 & 3 \\
\hline 29 & $\mathrm{n}$ & 0 & 2 & 0 & 2 \\
\hline 34 & $\mathrm{n}$ & 0 & 0 & 2 & 2 \\
\hline 11 & $\mathrm{n}$ & 0 & 1 & 0 & 1 \\
\hline 22 & $\mathrm{n}$ & 0 & 1 & 0 & 1 \\
\hline 23 & $\mathrm{n}$ & 0 & 0 & 1 & 1 \\
\hline 72 & $\mathrm{n}$ & 0 & 0 & 1 & 1 \\
\hline 92 & $\mathrm{n}$ & 0 & 1 & 0 & 1 \\
\hline \multicolumn{2}{|c|}{ Puntaje de ansiedad } & 56.95 & 41.84 & 29.95 & 41.95 \\
\hline \multicolumn{2}{|c|}{$\mathrm{n}$} & 216 & 317 & 267 & 800 \\
\hline
\end{tabular}


Según la especialidad a la que postulan, hemos encontrado que los mayores niveles de ansiedad ante los exámenes se encuentran en los postulantes a Medicina Humana, Derecho y Administración. En la especialidad de Medicina Humana han sido evaluados 119 estudiantes, de los cuales fueron diagnosticados con Ansiedad alta ante los exámenes 28 estudiantes, 56 con ansiedad moderada y 35 con ansiedad baja ante los exámenes. En la especialidad de derecho han sido evaluados 56 estudiantes; de los cuales fueron diagnosticados con ansiedad alta ante los exámenes 19 estudiantes, 26 con ansiedad moderada y 11 con ansiedad baja ante los exámenes. asimismo, en la especialidad de administración fueron evaluados 53 estudiantes; de los cuales fueron diagnosticados con ansiedad alta ante los exámenes 14 estudiantes, 26 con ansiedad moderada y 13 con ansiedad baja ante los exámenes.

La media muestral es de 41.92 puntos; tratándose de una investigación descriptiva, se ha considerado que la media poblacional oscila entre 41.19 y 42.69 que corresponden al diagnóstico de ansiedad moderada ante los exámenes. Asimismo, para referencia se adjuntan los estadísticos descriptivos obtenidos, los cuales se evidencian para mayor detalle en la tabla 4.

Tabla 4. Resultados estadísticos descriptivos.

\begin{tabular}{cc}
\hline Puntaje total & \\
\hline Media & 41.92 \\
Error típico & 0.40 \\
Mediana & 40.50 \\
Desviación estándar & 32.00 \\
Varianza de la muestra & 11.37 \\
Curtosis & 129.22 \\
Coeficiente de asimetría & -0.50 \\
Rango & 0.42 \\
Mínimo & 58.00 \\
Máximo & 17.00 \\
Suma & 75.00 \\
Cuenta & 33560.00 \\
Nivel de confianza & 800.00 \\
\hline
\end{tabular}

La variable ansiedad ante los exámenes está conformada por dos factores: emocionalidad y preocupación. El diagnóstico de estos factores nos indica una ligera predominancia del factor preocupación (el $29.13 \%$ de los estudiantes poseen un alto nivel de preocupación ante los exámenes) sobre el factor emocionalidad (el $28.50 \%$ de los estudiantes poseen un alto componente de emocionalidad); tal como puede apreciarse en la tabla 5 y la tabla 6. 
Tabla 5. Niveles del factor emocionalidad.

\begin{tabular}{lccc}
\hline \multicolumn{1}{c}{ Dx factor emocionalidad } & Promedio & $\mathrm{n}$ & Porcentaje \\
\cline { 2 - 4 } Componente emocional alto & 11.77 & 228 & 28.50 \\
Componente emocional moderado & 7.36 & 424 & 18.50 \\
Componente emocional bajo & 4.64 & 148 & 53.00 \\
\hline Total general & 8.11 & 800 & 100.00 \\
\hline
\end{tabular}

Tabla 6. Niveles del factor preocupación.

\begin{tabular}{cccc}
\hline Dx factor preocupación & Promedio & $\mathrm{n}$ & Porcentaje \\
\cline { 2 - 4 } Preocupación alta & 12.50 & 233 & 29.13 \\
Preocupación moderada & 8.91 & 281 & 35.13 \\
Preocupación baja & 5.98 & 286 & 35.75 \\
\hline Total general & 8.91 & 800 & 100.00 \\
\hline
\end{tabular}

Al efectuar el análisis de ítems encontramos que, en forma general, los mayores problemas se encuentran en los ítems 01, 10 y 12 (ver tabla 7) referidos a tensión durante el examen, inquietud antes de recibir el resultado del examen y confianza y tranquilidad al contestar el examen.

Tabla 7. Análisis general de ítems.

\begin{tabular}{ccc}
\hline & \multicolumn{2}{c}{ Total } \\
\cline { 2 - 3 } 01 & Promedio & DT \\
\cline { 2 - 3 } 02 & 2,49 & 0,80 \\
03 & 2,28 & 0,90 \\
04 & 2,21 & 0,98 \\
05 & 1,81 & 0,95 \\
06 & 1,84 & 0,97 \\
07 & 1,94 & 0,89 \\
08 & 2,26 & 0,94 \\
09 & 1,80 & 0,88 \\
10 & 2,17 & 0,91 \\
11 & 2,64 & 1,00 \\
12 & 2,17 & 0,90 \\
13 & 2,82 & 1,11 \\
& 1,61 & 0,90
\end{tabular}




\begin{tabular}{lll}
14 & 1,61 & 0,82 \\
15 & 1,65 & 0,87 \\
16 & 2,42 & 0,94 \\
17 & 2,38 & 1,02 \\
18 & 1,86 & 0,90 \\
19 & 2,15 & 0,94 \\
20 & 2,16 & 0,93 \\
\hline
\end{tabular}

Al analizar los ítems considerando la variable sexo, encontramos que, tanto los varones como las mujeres, presentan mayores problemas en los ítems referidos a tensión durante el examen e inquietud antes de recibir el resultado del examen (ver tabla 8).

Tabla 8. Análisis de ítems según sexo.

\begin{tabular}{|c|c|c|c|c|}
\hline \multirow[t]{2}{*}{ Ítem } & \multicolumn{2}{|c|}{ Varones } & \multicolumn{2}{|c|}{ Mujeres } \\
\hline & Promedio & DT & Promedio & DT \\
\hline 01 & 2.36 & 0,84 & 2,61 & 0,76 \\
\hline 02 & 2.17 & 0,88 & 2,38 & 0,90 \\
\hline 03 & 2.11 & 0,98 & 2,30 & 0,97 \\
\hline 04 & 1.63 & 0,87 & 1,97 & 0,98 \\
\hline 05 & 1.79 & 0,95 & 1,89 & 0,99 \\
\hline 06 & 1.91 & 0,89 & 1,96 & 0,90 \\
\hline 07 & 2.15 & 0,92 & 2,36 & 0,94 \\
\hline 08 & 1.78 & 0,86 & 1,82 & 0,89 \\
\hline 09 & 2.07 & 0,87 & 2,26 & 0,93 \\
\hline 10 & 2.51 & 1,02 & 2,75 & 0,98 \\
\hline 11 & 2.00 & 0,85 & 2,33 & 0,92 \\
\hline 12 & 2.68 & 1,13 & 2,95 & 1,08 \\
\hline 13 & 1.46 & 0,76 & 1,74 & 0,99 \\
\hline 14 & 1.55 & 0,78 & 1,66 & 0,85 \\
\hline 15 & 1.50 & 0,76 & 1,78 & 0,94 \\
\hline 16 & 2.25 & 0,91 & 2,58 & 0,93 \\
\hline 17 & 2.26 & 0,99 & 2,49 & 1,04 \\
\hline 18 & 1.73 & 0,82 & 1,97 & 0,95 \\
\hline 19 & 1.99 & 0,88 & 2,28 & 0,97 \\
\hline 20 & 2.00 & 0,90 & 2,30 & 0,94 \\
\hline
\end{tabular}




\section{DISCUSIÓN}

Los resultados obtenidos corroboran la alta incidencia de la ansiedad en situación de examen, con un mayor nivel de ansiedad ante los exámenes en los estudiantes del sexo femenino.

\section{CONCLUSIONES}

1. El $27 \%$ de los estudiantes del CEPRE-UNMSM presentan ansiedad alta ante los exámenes. Mientras que el $33.38 \%$ tienen ansiedad baja, el $39.63 \%$ ansiedad moderada ante los exámenes.

2. Los estudiantes del sexo femenino tienen en promedio mayor nivel de ansiedad ante los exámenes que sus pares del sexo masculino.

3. Las aulas que poseen mayor nivel de Ansiedad ante los exámenes son las aulas: 27, $19,06,20,37,13$ y 03.

4. Los mayores niveles de ansiedad se encuentran en los postulantes a Medicina Humana, Derecho y Administración.

5. Los resultados indican que existe una ligera predominancia del factor preocupación ante los exámenes que el factor emocionalidad.

6. Los mayores problemas se observan en los ítems referidos a confianza y tranquilidad ante el examen, inquietud antes de recibir el resultado del examen y tensión digestiva durante el examen.

7. Existe una serie de variables personales, familiares, socioeconómicas y culturales que coadyuvan a intensificar esta problemática; razón por la cual es necesario reseñar las conclusiones obtenidas a través de la atención psicopedagógica a fin de que sirvan de base para planificar futuras acciones correctivas.

\section{Conclusiones obtenidas de la entrevista personal}

Considerando las evaluaciones personales realizadas cuantitativamente como cualitativamente en la entrevista personal, a la mayoría de los casos atendidos en razón a la ansiedad ante los exámenes se le encontraron indistintamente las siguientes características:

1. Tienen presión de los padres a desarrollar una carrera que no siempre es de su vocación.

2. Insistencia del familiar o familiares a que ya ingrese. Son comparados a nivel familiar con otros parientes que ya estudian en la universidad.

3. Bajo rendimiento académico. Deserción por ignorar materias, lo que impide continuar con la secuencia de los cursos. Se ha encontrado que muchos alumnos no han tenido base necesaria para manejar cognitivamente los diferentes cursos que se imparten en la pre, especialmente en los de ciencias.

4. Problemas familiares. Familias disfuncionales. Padres separados. 
5. Poco control de emociones. Problemas de autoestima. Predisposición al estrés, ansiedad o depresión. Están pendiente del qué dirán.

6. Un buen grupo son de provincias, viven solos y tienen nostalgia de su familia.

7. Frustración por todavía no ingresar (en especial en aquellos que vienen desarrollando varios ciclos) y por la expectativa que manejaron subjetivamente de ingresar a la universidad fácilmente por haber estado en el cuadro de mérito de sus colegios.

8. Trastornos psicosomáticos: gastritis, cefaleas, alteración del sudor, insomnio, falta de apetito, retrasos menstruales, etc.

9. Problemas asociados a situaciones reactivas. Pérdida de trabajo del padre o fallecimiento de un familiar.

10. Alteraciones de personalidad tendiendo a la introversión, acciones obsesivo-compulsivas, bipolaridad, reacciones neuróticas, etc.

11. No tienen sistema en hábitos de estudios o es deficiente. Caen fácilmente en el desorden, por no obligarse orden y disciplina. Usan el tiempo morosamente, estudiando a última hora.

12. No manejan estrategias de aprendizaje óptimas. Problemas de memoria. Déficit de atención o concentración.

13. Hay carencias económicas. Hacinamiento familiar o habitacional.

14. Incongruencia del sentido de realidad, manejando prejuicios a nivel personal o social.

\section{Estrategias de atención empleadas}

Asesoría y consejería, además de la aplicación de psicoterapias breves en:

1. El mejoramiento de la autoestima.

2. Control de impulsos o emociones.

3. Optimización de la memoria.

4. Técnicas de mejoramiento en atención y concentración.

5. Hábitos de estudio.

6. Uso del tiempo.

7. Concientización de su realidad.

8. Estrategias de aprendizaje.

9. Importancia del orden y la disciplina en el éxito.

10. Control de conductas neuróticas o hiperactivas.

11. Regulación de sus necesidades homeostáticas base, hambre, sed, sueño.

12. La importancia del descanso cuando estudian. 
13. La motivación personal y la vocación profesional.

14. Inteligencia emocional.

15. Regulación del estrés, ansiedad o depresión.

16. Otros.

\section{Alcances}

Alumnos.

Padres de familia.

Auxiliares.

Profesores.

\section{Conclusiones finales}

1. La muestra de 800 alumnos en todas las sedes ha sido representativa para la investigación.

2. La problemática detectada constituye una situación generalizada, la cual debe atenderse a tiempo, ya que en muchos casos viene condicionándose desde las escuelas y se profundiza en esta fase preparatoria. Especialmente porque para muchos alumnos esta es su primera experiencia en el CEPRE-UNMSM.

3. Pasa a ser también un problema psicosocial, por lo que muchas de sus determinantes tienen que ver con las relaciones familiares del mismo núcleo, que se hacen extensivas hacia otros familiares y al entorno social en general.

4. La alta tasa de ansiedad encontrada ha generado en muchos de los alumnos alteraciones psicosomáticas, con mayor presencia de gastritis, insomnio y pérdida de apetito.

\section{REFERENCIAS BIBLIOGRÁFICAS}

Alcalde Lapiedra, M. T. (1991). Los trastornos de conducta en la infancia y sus relaciones con las vivencias de ansiedad y depresión. Zaragoza: Universidad.

Ayuso Gutiérrez, J. L. (1988). Trastornos de angustia. Barcelona: Martínez Roca.

Cano-Vindel, A. (1989). Cognición, emoción y personalidad: un estudio centrado en la ansiedad. Madrid: Universidad Complutense.

Cano-Vindel, A. (2002). La ansiedad. Claves para vencerla. Málaga: Arguval.

Cano-Vindel, A., \& Miguel-Tobal, J. J. (1990). Differences between healthy and psychosomatic subjects in the pattern of anxiety responses in different types of situations. En C.O.P. (Ed.), Psicología y Salud: Psicología de la Salud (pp. 62-67). Madrid: Colegio Oficial de Psicólogos (COP). 
Chávez, E. (2004). Estrategias de afrontamiento a la ansiedad de evaluación y su relación con el desempeño académico en estudiantes universitarios incorporados a modelos educativos innovadores (Tesis de Maestría, Universidad de Colima).

Escalona, A. (1999). Ansiedad y rendimiento: intervención cognitivo-conductual para la reducción de la ansiedad ante los exámenes y la mejora del rendimiento académico. Madrid: Universidad Complutense de Madrid (tesis doctoral).

Eysenck, M.W. y Derakshan, N. (1997). Un marco teórico cognitivo para los trastornos de ansiedad. Ansiedad y Estrés, 3, 121-134 (Monografía 'Técnicas de reducción de ansiedad')

Gamboa, J. (2010). Efecto de un programa de biodanza en un grupo de estudiantes de la carrera de Psicología con síntomas ansiosos. Revista electrónica de Psicología Iztacala. Consultado en Marzo, 04, 2012 en http://www.iztacala.unam.mx/carreras/ psicologia/psiclin/vol13num4/Vol13No4Art9.pdf.

Grandis, A. (2009). Evaluación de la ansiedad frente a los exámenes universitarios (Tesis de Doctorado, Universidad Nacional de Córdoba).

Gutiérrez, M. y García, M. (1997). Ansiedad y Cognición: Un marco integrador. Revista Electrónica de Motivación y Emoción. Consultado en Marzo, 04, 2012 en http:// reme.uji.es/articulos/agutim660131299/texto.html.

Piemontesi, S., Heredia, D. y Furián, L. (2009, № 2. pp 74 - 86). Correlatos de la ansiedad ante los exámenes: Una aproximación a la teoría de la reducción en la eficiencia. Revista Tesis. Consultado en Marzo, 02, 2012 en http://revistas.unc.edu.ar/index. php/tesis/article/viewFile/292/pdf_14.

Reyes, Y. (2003). Relación entre el rendimiento académico, la ansiedad ante los exámenes, los rasgos de personalidad, el autoconcepto y la asertividad en estudiantes del primer año de Psicología de la UNMSM (Tesis de Licenciatura, UNMSM).

Valero, L. (1999). Evaluación de ansiedad ante exámenes: Datos de aplicación y fiabilidad de un cuestionario CAEX. Anales de Psicología. Consultado en Marzo, 04/2012 en http://revistas.um.es/analesps/article/view/30101. 